\title{
Mobile Versuchsanlage zur Ertragssteigerung und Prozessoptimierung von Biogasanlagen
}

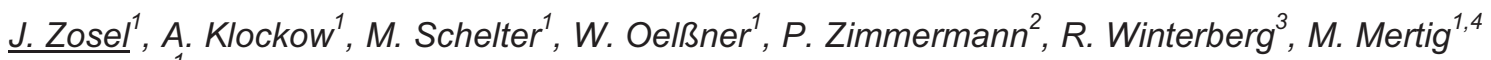 \\ ${ }^{1}$ Kurt-Schwabe-Institut für Mess- und Sensortechnik e.V. Meinsberg (KSI), \\ Kurt-Schwabe-Straße 4, D-04736 Waldheim, zosel@ksi-meinsberg.de, Deutschland \\ ${ }^{2}$ TEB Ingenieurbüro P. Zimmermann, Mühltaler Straße 6, D-12555 Berlin, Deutschland \\ ${ }^{3}$ Cordes + Winterberg GbR, Seestraße 2A, D-39175 Biederitz, Deutschland \\ ${ }^{4}$ Technische Universität Dresden, Professur für Physikalische Chemie, Mess- und Sensortechnik
}

\begin{abstract}
Zusammenfassung:
Zur Ertragssteigerung und Prozessoptimierung von Biogasanlagen wurde eine mobile Versuchsanlage entwickelt, hergestellt und in einem Langzeit-Feldversuch erprobt. Der in einem Container untergebrachte Fermenter mit $1,5 \mathrm{~m}^{3}$ Rauminhalt enthält mehrere neu entwickelte Sensoren zur Beurteilung des Prozesszustandes sowie eine Vorrichtung zur Erkennung und Bekämpfung von Schaum. Während des Feldversuches konnte eindeutig nachgewiesen werden, dass sich Störeinflüsse auf die Gelöst-Wasserstoff-Konzentration auswirken, die mit höherer Aussagekraft als die Wasserstoff-Konzentration im Biogas als Stabilitätskriterium herangezogen werden kann. Während einer kritischen Substratumstellung konnten weiterhin Zuverlässigkeit und Effektivität des Sensor/AktorSystems zur Schaumbekämpfung im Headspace des Fermenters unter Beweis gestellt werden.
\end{abstract}

Schlüsselwörter: mobile Biogasanlage, Multisensorik, Gelöst-Wasserstoff-Messung, Schaum

\section{Problemstellung}

Mit unterschiedlichen und wechselnden Substraten sowie biogenen Reststoffen betriebene Biogasanlagen zeigen häufig instabiles $\mathrm{Be}$ triebsverhalten und werden deshalb oft weit unterhalb ihres möglichen Leistungspotenzials betrieben [1]. In vielen Fällen ist die Ursache dafür unzureichendes Prozess-Monitoring. Einschlägige Studien und eigene Arbeiten haben deutlich gezeigt, dass der aktuelle Anlagenbestand nur bedingt für die Nutzung dieser Substrate geeignet ist. Vor allem ist das Fermentationsvolumen zahlreicher großtechnischer Anlagen in vielen Fällen zu gering für die Nutzung problematischer oder langsam abbaubarer Substrate (Festmist, Hühnertrockenkot, Hirseund Grassilage). Bei den meisten der in Deutschland vorhandenen Biogasanlagen besteht deshalb ein Optimierungspotenzial von mindestens $10 \%$. Mittels der hier vorgestellten mobilen Biogas-Versuchsanlage "VESBA" soll dieses Potenzial erschlossen werden. Dabei werden folgende Ziele verfolgt:

- Komplexe Erfassung des Ist-Zustandes,

- Optimierung der Substratvorbehandlung,

- Verbesserung der Prozesseffizienz,

- Erhöhung des Methanertrags,

- Vermeidung toxischer Effekte sowie

- Repowering der Anlage.

\section{Lösungsansatz}

Die mobile Biogas-Versuchsanlage VESBA ist mit Vorrichtungen zur Fütterung mit festen und flüssigen Substraten ausgestattet. Viele, teilweise neu entwickelte Sensoren im Gärmedium und im Biogas ermöglichen die sichere und schnelle Erfassung des Zustandes des Biogasmediums. Ein neues Messsystem zur Insitu-Bestimmung des gelösten Wasserstoffs im flüssigen Inhalt des Fermenters [2] liefert in Echtzeit Schlüsselinformationen, die als Basis für abzuleitende verfahrenstechnische Maßnahmen hinsichtlich der Prozessoptimierung sowie auch der Erkennung von Belastungsgrenzen zur sicheren Vermeidung von Systemabstürzen geeignet sind.

Ein wesentliches Entwicklungsziel des VESBAProjekts war, den sicheren Anlagenbetrieb im Feldeinsatz mit minimalem Personalaufwand zu gewährleisten. Als sinnvolle Lösung erweist sich hierfür, die Anlage durch den Aufbau einer stabilen Datenverbindung über das Mobilfunknetz für Berechtigte kontrollierbar zu machen. Auf diese Weise wird die permanente Anlagenüberwachung bei gegebener Möglichkeit, direkt auf die Steuerung einzuwirken, realisiert. Zusätzlich wird durch eine mehrstufige Übertragung der Messdaten auch bei Hardwareausfall eine hohe Datensicherheit erzielt. 

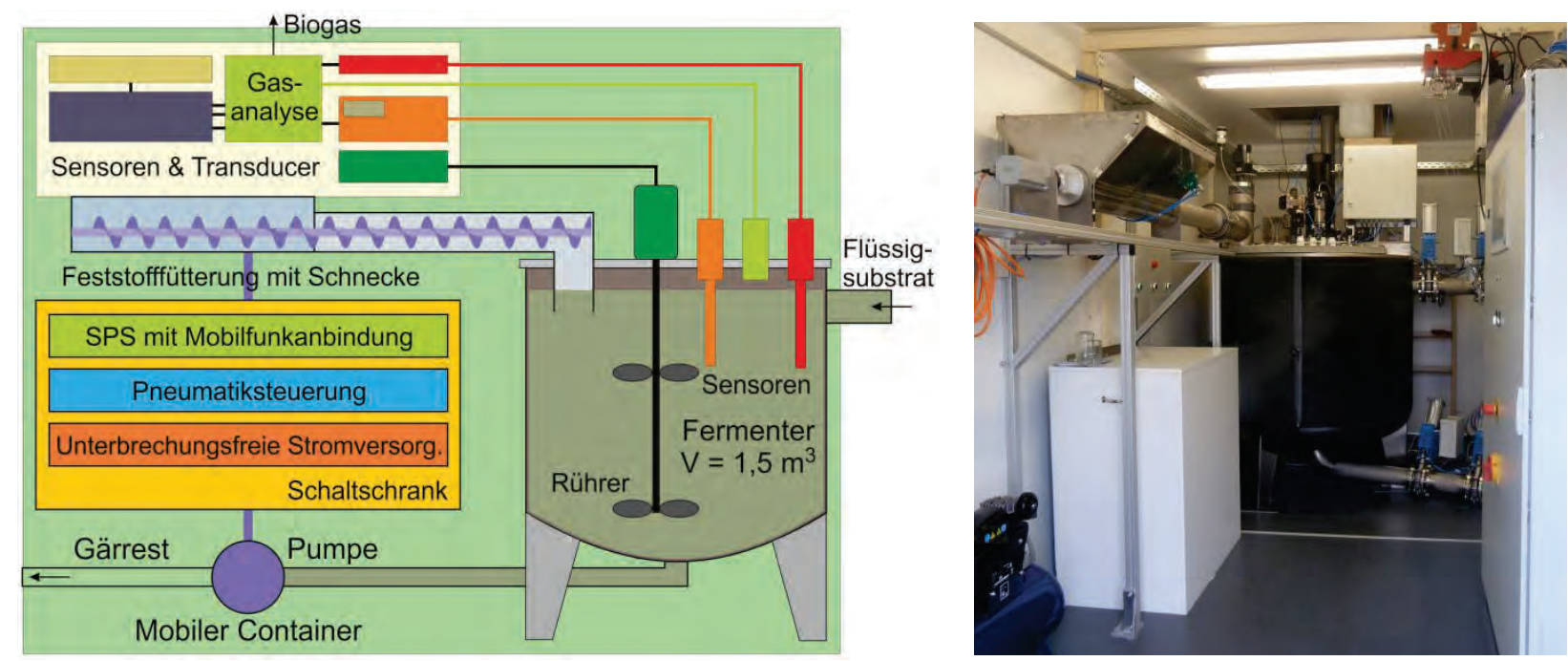

Bild 1: Schema und Innenansicht der portablen Biogas-Anlage "VESBA" mit verschiedenen Möglichkeiten der Feststoff- und Flüssigfütterung

\section{Technische Realisierung}

Die in Bild 1 gezeigte mobile Biogasanlage ist in einem transportablen Container untergebracht. Sie enthält einen zylindrischen Fermenter mit 1,5 $\mathrm{m}^{3}$ Fassungsvermögen, der durch ein Rohr- und Pumpsystem an eine kommerzielle Biogasanlage gekoppelt werden kann.

Zur Fütterung fester Substrate wurden automatische Fütterungseinrichtungen entwickelt. Insbesondere wurden neue Lösungen gefunden, um dem vergleichsweise kleinen Fermenter auch sehr grobstückige Substrate unter Sauerstoffabschluss quasi-kontinuierlich zuführen zu können, wobei nur einmal täglich Substrat nachgefüllt werden muss. Die Biogasanlage verfügt außerdem über einem weiteren Vorratsbehälter mit Dosiervorrichtung zur automatischen Fütterung flüssiger Substrate.

Die in Tabelle 1 aufgeführten Sensoren sorgen für die sichere und schnelle Erfassung des Zustandes des Biogasmediums.
Das neu entwickelte In-Situ-Messsystem zur Bestimmung des gelösten Wasserstoffs besteht im Wesentlichen aus einer Extraktionseinheit, einem Gaschromatographen und einer Festelektrolyt-Zelle, die als coulometrischer Detektor eingesetzt wird [2].

Mittels einer speicherprogrammierbaren Steuerung (WAGO-SPS) wurden Automatisierung, Signalverarbeitung und Datenaufzeichnung der mobilen Biogasanlage implementiert. Die Visualisierung aller Parameter und die Bedienung von manuell ansprechbaren Komponenten erfolgt über einen Kontrollrechner mit berührungssensitivem Monitor.

Die Anlage wird bei Ausfällen oder Störungen an wichtigen Aggregaten automatisch in sichere Betriebszustände überführt, wobei berechtigte Personen durch eine kurze Fehlermitteilung per Mobilfunk darüber informiert werden. Diese Übertragung wird außerdem zur Anlagensteuerung sowie für den Messdatentransfer genutzt und eröffnet somit die Möglichkeit der effektiven Fernüberwachung der Anlage.

Tab. 1: Übersicht über die in der mobilen Biogasanlage enthaltenen Sensoren

\begin{tabular}{|l|l|}
\hline Sensor & Messgröße \\
\hline Extraktionseinheit mit GC & gelöster Wasserstoff \\
\hline Glaselektrode & pH-Wert \\
\hline Redoxelektrode & Redoxpotenzial \\
\hline Referenzelektrode & Bezugspotenzial für pH- und Redox-Messung \\
\hline Infrarot-Gassensor & Methankonzentration \\
\cline { 2 - 2 } & Kohlendioxidkonzentration \\
\hline Amperometrischer Gassensor & Wasserstoffkonzentration \\
\hline Thermischer Massestromsensor & Biogas-Volumenstrom \\
\hline Drucksensoren (3 Stück) & Füllstand und Druck im Headspace \\
\hline Leitfähigkeitssensor & Schaumerkennung \\
\hline PT-1000-Sensor & Fermenter-Innentemperatur \\
\hline PT-100-Sensoren (4 Stück) & Heizungstemperaturen \\
\hline PT-100-Sensoren (6 Stück) & Anlagentemperaturen \\
\hline
\end{tabular}




\section{Ergebnisse}

Zur Untersuchung wesentlicher Eigenschaften der VESBA wurde ein Langzeitversuch an einer Biogas-Großanlage durchgeführt, die mit einem Substratgemisch aus Maissilage, Hühnertrockenkot (HTK) und Rindergülle (48/27/25 Masse-\%) gefüttert wird. Zugrunde lag die Fragestellung, auf welche Weise und in welchem Ausmaß sich ohne Prozessbeeinträchtigung der HTK-Anteil erhöhen lässt. Der Fermenter der VESBA wurde zu Beginn mit Gärsubstrat befüllt, das unmittelbar zuvor aus der BiogasGroßanlage entnommen worden war. Mit der Fütterung des o.g. Substratgemisches wurde sofort nach der Befüllung begonnen. Nach einer Startphase mit konstanter Fütterung der Anteile wurde der HTK-Anteil zugunsten der Maissilage kontinuierlich auf etwa 40 Masse-\% erhöht und eine weitere Stabilisierungsphase abgewartet.

Während der Startphase wurden die Betriebsparameter der VESBA an das Substratgemisch angepasst und der Prozess optimiert. Eine Anpassung betraf die Fermenterheizung, die gemäß Bild 2 aus 4 flexiblen und getrennt ansteuerbaren Heizmatten bestand, die auf die $2 \mathrm{~mm}$ dicke Edelstahl-Wandung des Fermenters aufgeklebt waren. Der Temperaturregelung lag zunächst ein Zweipunkt-Heizer mit einer Hysterese $>20^{\circ} \mathrm{C}$ zugrunde, wobei im eingeschwungenen Zustand während einer Heizphase die vier Heizmatten einzeln im Umlaufbetrieb angesprochen wurden (Bild 2). Der sich dadurch für das wandnahe Substrat ergebende thermische Stress während der Heizphase bildete sich mit überraschend kurzer Ansprechzeit sowohl in den aus dem Biogas gewonnenen Signalen als auch bei der Gelöst- $\mathrm{H}_{2}$-Konzentration ab, wie die in Bild 3 dargestellten Kurven belegen.

Neben dem gut sichtbaren sofortigen steilen Anstieg von Methan- und Wasserstoffvolumenstrom im Biogas zeigt sich ein zeitlich verzögerter zweiter Anstieg, der durch die steigende Temperatur im gesamten Substrat hervorgerufen wird. Der zeitlich verzögerte Anstieg des Signals des Gelöst- $\mathrm{H}_{2}$-Sensors nach einer Heizperiode ist auf dessen Positionierung in der
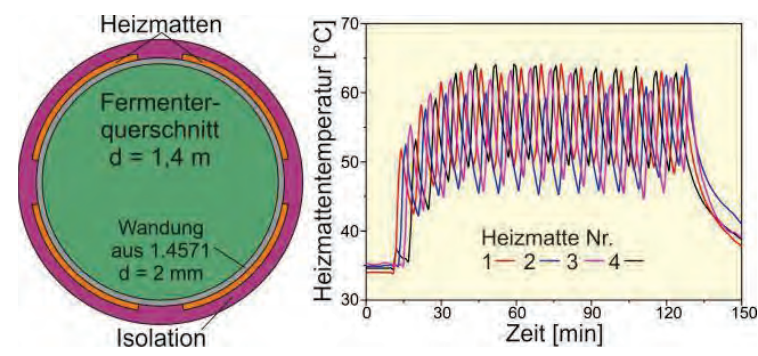

Bild 2: Fermenterheizung und typischer Temperaturverlauf an den Heizmatten während einer Heizphase bei nicht optimierter Zweipunktregelung

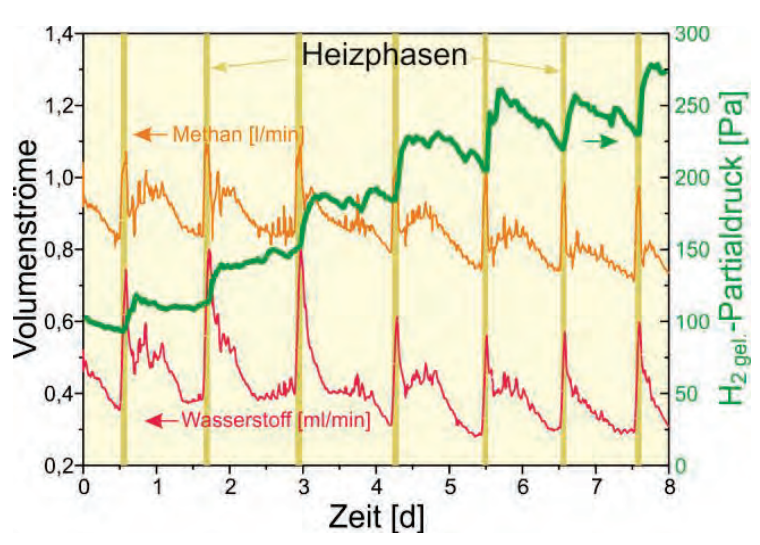

Bild 3: Verlauf von Gelöst- $\mathrm{H}_{2}$-Partialdruck sowie Methan- und $\mathrm{H}_{2}$-Volumenstrom im Biogas während der Startphase bei Zweipunkt-Regelung der Heizung

Fermentermitte zurückzuführen, da der Thermopuls hier deutlich später wirksam wird.

Der Vergleich zwischen dem Gelöst- $\mathrm{H}_{2}$-Partialdruck und dem $\mathrm{H}_{2}$-Volumenstrom im Biogas während des in Bild 3 gezeigten Teils der Startphase belegt, dass sich im Verlauf einer Woche der Wasserstoff nur im Substrat zu akkumulieren scheint. Dieses Phänomen kann gemäß Literatur [3] u.a. darauf zurückgeführt werden, dass sich im Headspace von Biogas-Anlagen bereits nach wenigen Tagen Bakterienkulturen ansiedeln können, die den gasförmig emittierten Wasserstoff gemeinsam mit Leckage-Sauerstoff als Energiequelle nutzen. Der Kurvenverlauf ist ein Indiz für diese Ursache.

Nachdem die Heizungsregelung so optimiert wurde, dass die pro Heizvorgang lokal eingetragene Energiemenge ein Minimum annimmt, sank der Gelöst- $\mathrm{H}_{2}-$ Partialdruck sofort deutlich $a b$, wie die Resultate in Bild 4 belegen. Damit war ein stabiler Anlagenbetrieb gesichert, und die Methanproduktion erhöhte sich bis zum Beginn der Steigerung des HTK-Anteils.

Zu Beginn dieser Steigerungsphase des HTKAnteils ging die Methanbildung deutlich zurück und mündete in ein Minimum nach etwa 48 Versuchstagen. Dieser Rückgang ging einher mit einem deutlichen Anstieg der Viskosität des Gärmediums und war außerdem durch eine massive Schaumbildung gekennzeichnet. Nach der Installation einer Schaumbekämpfung stabilisierte sich der Prozess wieder. Die verringerten Methanvolumenströme zum Ende der HTK-Steigerungsphase sind auf verringerte Fütterungsraten der festen Substrate zurückzuführen. Diese werden u.a. durch eine abnehmende Förderfähigkeit des Maissilage/HTKGemisches im installierten Schneckenförderer verursacht, da HTK die Anhaftung des Substrates an den Behälterwänden sowie die Brückenbildung verstärkt. 


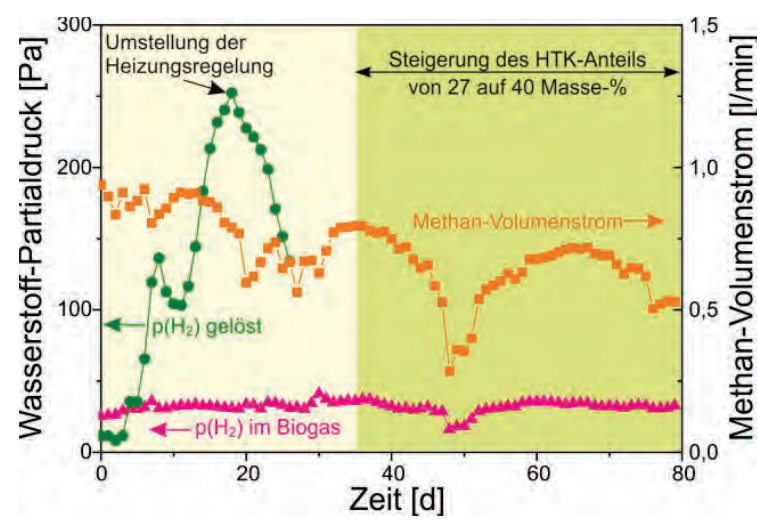

Bild 4: Verlauf von Methan-Volumenstrom und Wasserstoffpartialdrücken im Biogas sowie im Substrat während des Langzeitversuches neben einer BiogasGroßanlage

Wie oben bereits angedeutet, muss für eine zuverlässige Prozessführung insbesondere bei kritischen Substratumstellungen ein System zur Schaumerkennung und -bekämpfung im Biogasfermenter unbedingt vorhanden sein, da die Schaumbildung ein frühes Gefahrenzeichen für Prozessinstabilitäten ist. Um bislang ungelöste Probleme bei der Schaumbekämpfung in Labor-Biogasfermentern zu umgehen, wurde das in Bild 5 aufgeführte Sensor/Aktor-System neu entwickelt und erprobt. Es besteht aus zwei Edelstahl-Leitfähigkeitselektroden und einem Perfusor mit Pralldüse, durch den impulsartig flüssige Medien zur Schaumbekämpfung vernebelt werden. Bei Auftreten von Schaum (Schaumsignal > $10 \%$ ) wurden pro Stunde durch die Pralldüse 15 Druckimpulse von je $20 \mathrm{ml}$ Wasser abgegeben. Das Design der Pralldüse und die fluiddynamische Auslegung ermöglichten eine scheibenförmige Vernebelung des Wassers im Headspace mit einem Durchmesser von etwa $60 \mathrm{~cm}$. Im Bereich dieser Vernebelung waren alle weiteren Sensoren positioniert, so dass der Prozess auch bei Schaumbildung ohne Unterbrechung weitergeführt werden konnte.

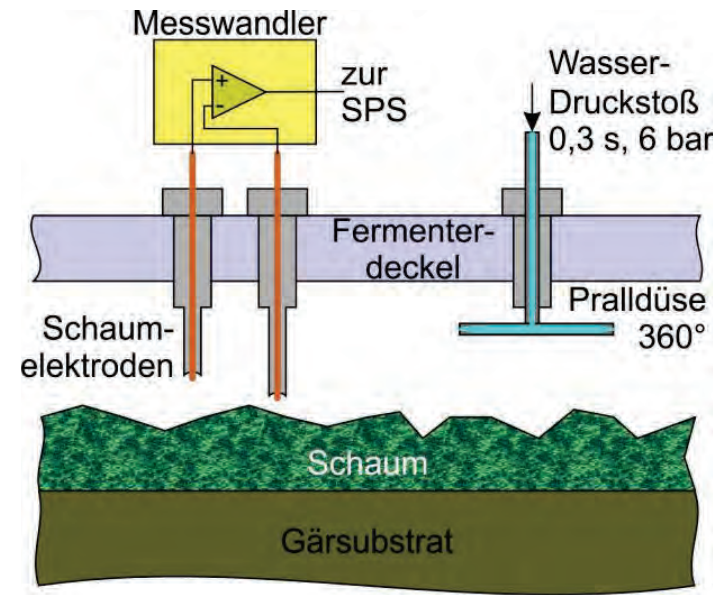

Bild 5: Sensor/Aktor-System zur Erkennung und Bekämpfung von Schaum
Der in Bild 6 gezeigte Tagesverlauf des vom Messwandler gemäß Bild 5 erhaltenen Ausgangssignals ist für das Auftreten von Schaum typisch. Die Wirksamkeit dieses Sensor/Aktor-Systems konnte über mehrere Wochen Prozessdauer mit Schaumbildung demonstriert werden.

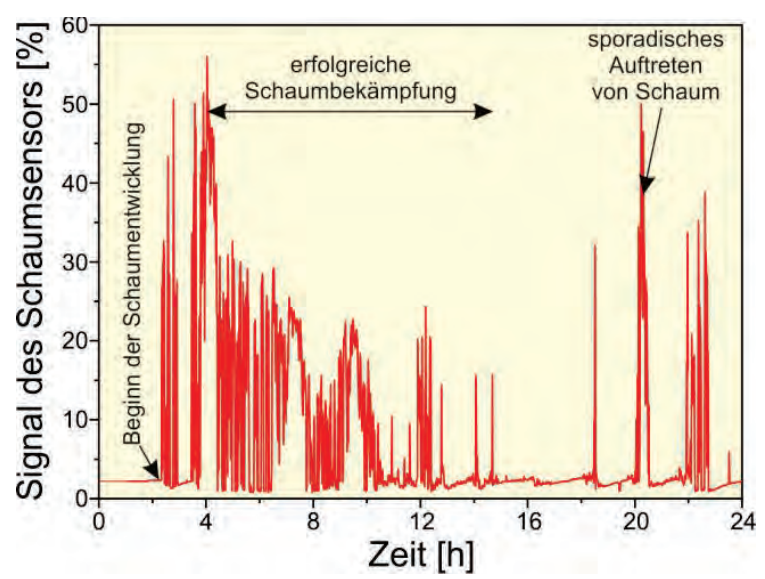

Bild 6: Typischer Tagesverlauf des Signals des Schaumsensors während der Phase der HTK-Steigerung

\section{Schlussfolgerung}

Mobile Biogas-Versuchsanlagen schaffen völlig neue Möglichkeiten, bestehende Biogasanlagen zu optimieren und weitere Biogaspotenziale zu erschließen. Die hier vorgestellte Anlage ist ein flexibel einsetzbares Werkzeug, das insbesondere für die Beurteilung von Anlagen zur Verwertung biogener Rest- und Abfallstoffe hervorragend geeignet ist. Die gewonnenen wissenschaftlichen Erkenntnisse zur Technologie und Dynamik von Biogasprozessen sollen auf reale Biogasanlagen übertragen werden.

\section{Danksagung}

Das dieser Arbeit zugrunde liegende Vorhaben wurde mit Mitteln des Bundesministeriums für Umwelt, Naturschutz und Reaktorsicherheit (BMU) unter dem Förderkennzeichen 03KB045A gefördert. Die Verantwortung für den Inhalt dieser Arbeit liegt bei den Autoren, die für die Förderung danken.

\section{Literatur}

[1] Fachagentur Nachwachsende Rohstoffe e.V. (FNR): Biogas-Messprogramm II.

[2] M. Schelter, et al., A solid electrolyte sensor for trace gas analysis, Sensors and Actuators B: Chemical 187, 209-214 (2013); doi: 10.1016/j.snb.2012.10.111

[3] A. Pauss, et al., Liquid-to-Gas Mass Transfer in Anaerobic Processes: Inevitable Transfer Limitations of Methane and Hydrogen in the Biomethanation Process, Appl. Environ. Microb., 56 1636-1644 (1990) 